\title{
Inclusive production of charged pion pairs in proton-antiproton collisions
}

\author{
A. I. Ahmadov,,$^{1, *}$ C. Aydin, ${ }^{2, \dagger}$ and O. Uzun ${ }^{2, *}$ \\ ${ }^{1}$ Department of Theoretical Physics, Baku State University, Z. Khalilov st. 23, AZ-1148, Baku, Azerbaijan \\ ${ }^{2}$ Department of Physics, Karadeniz Technical University, 61080, Trabzon, Turkey
}

(Received 11 August 2018; revised manuscript received 19 December 2018; published 11 March 2019)

\begin{abstract}
In this study, we have considered the contribution of the higher-twist (HT) effects of the subprocesses to inclusive pion pair production cross section in the high energy proton-antiproton collisions by using various pion distribution amplitudes (DAs) within the frozen coupling constant approach and compared them with the leading-twist contributions. The feature of the HT effects may help the theoretical interpretation of the future PANDA experiment. The dependencies of the HT contribution on the transverse momentum $p_{T}$, the center of mass energy $\sqrt{s}$, and the variable $x_{T}$ are discussed numerically with special emphasis put on DAs. Moreover, the obtained analytical and numerical results for the differential cross section of the pion pair production are compared with the elastic backward scattering of the pion on the proton. We show that the main contribution to the inclusive cross section comes from the HT direct production process via gluon-gluon fusion. Also, it is strongly dependent on the pion DAs, momentum cut-off parameter $\Delta p$, and $\left\langle q_{T}^{2}\right\rangle$ which is the mean square of the intrinsic momentum of either initial parton.
\end{abstract}

DOI: 10.1103/PhysRevC.99.035204

\section{INTRODUCTION}

It is well known that quantum chromodynamics (QCD) is the fundamental theory of strong interactions. QCD describes the strong interactions between quarks and gluons, also the structure and dynamics of hadrons at the amplitude level.

The hadronic distribution amplitude (DA) in terms of internal structure degrees of freedoms is important in QCD process predictions. Parton DAs are important ingredients in applying QCD to hard exclusive processes via the factorization theorem [1-3]. Understanding of the hadronic structure in terms of the fundamental degrees of freedom of QCD is one of the fascinating questions of the popular research area in physics. The important processes of the perturbative quantum chromodynamics (pQCD) are hadron pair production at large transverse momenta in hadron-hadron collisions. While parton distributions at leading twist (LT) are basically relevant to the description to the accuracy of leading power and refer to parton configurations with the minimal number of constituents. However, the higher-twist (HT) distributions are more numerous and they are used to consider the various effects owing to parton virtuality, transverse momentum, and

\footnotetext{
*ahmadovazar@yahoo.com

$\dagger$ coskun@ktu.edu.tr

†oguzhan_deu@hotmail.com
}

Published by the American Physical Society under the terms of the Creative Commons Attribution 4.0 International license. Further distribution of this work must maintain attribution to the author(s) and the published article's title, journal citation, and DOI. Funded by $S C O A P^{3}$. contributions from higher Fock states which are relevant to describe the power-suppressed corrections in the hard momentum. Braun et al. [4-6] recognized the important role of the LT and the HT parton distributions in hard exclusive process. The existing theoretical framework for the DA description is based on the conformal symmetry of the QCD Lagrangian for an exhaustive review [1-3,7-10].

The main difficulty in making precise perturbative QCD predictions is the uncertainty in determining the renormalization scale $\mu$ of the running coupling $\alpha_{s}\left(\mu^{2}\right)$. In practical calculations, it is difficult to guess a simple physical scale of the order of a typical momentum transfer in the process. Then we need to vary this scale over a range $Q / 2,2 Q$. In a common case, this problem for all orders was solved in Refs. [11,12]. Evolution kernels are the main tools of the well-known evolution equations for the parton distribution in deep inelastic scattering processes and for the parton distribution amplitudes in hard exclusive reactions. The DokshitzerGribov-Lipatov-Altarelli-Parisi (DGLAP) [13-16] equations describe the dependence of the parton distributions on the renormalization scale $\mu^{2}$. Until now, DGLAP evolution equations have been known as the most successful and major tools to study the structure functions of hadrons and ultimately structure of matter, ultra-high-energy cosmic rays. Also, the DGLAP equations describe the influence of the perturbative QCD corrections on the distribution functions that enter the parton model of deep inelastic scattering processes defined in the form as

$$
\frac{d}{d \ln \mu^{2}} G_{i}\left(x, \mu^{2}\right)=\frac{\alpha_{s}\left(\mu^{2}\right)}{2 \pi} \int_{x}^{1} \frac{d y}{y} G_{i}\left(y, \mu^{2}\right) P_{q q}\left(\frac{x}{y}\right) .
$$


From Eq. (1.1), we obtain an integrodifferential equation in the logarithm of the virtuality

$$
\frac{d}{d \ln \mu^{2}} x G\left(x, \mu^{2}\right)=\frac{\alpha_{s}}{2 \pi} \int_{x}^{1} d y\left[\sum_{f} P_{g q}\left(\frac{x}{y}\right)\left(\frac{x}{y} q_{f}\left(\frac{x}{y}, \mu^{2}\right)+\frac{x}{y} \bar{q}_{f}\left(\frac{x}{y}, \mu^{2}\right)\right)+P_{g g}\left(\frac{x}{y}\right) \frac{x}{y} G\left(\frac{x}{y}, \mu^{2}\right)\right] .
$$

Analogously, one finds the quark and antiquark distributions as

$$
\begin{aligned}
& \frac{d}{d \ln \mu^{2}} x q_{f}\left(x, \mu^{2}\right)=\frac{\alpha_{s}}{2 \pi} \int_{x}^{1} d y\left[P_{q q}\left(\frac{x}{y}\right) \frac{x}{y} q_{f}\left(\frac{x}{y}, \mu^{2}\right)+P_{q g}\left(\frac{x}{y}\right) \frac{x}{y} G\left(\frac{x}{y}, \mu^{2}\right)\right], \\
& \frac{d}{d \ln \mu^{2}} x \bar{q}_{f}\left(x, \mu^{2}\right)=\frac{\alpha_{s}}{2 \pi} \int_{x}^{1} d y\left[P_{q q}\left(\frac{x}{y}\right) \frac{x}{y} \bar{q}_{f}\left(\frac{x}{y}, \mu^{2}\right)+P_{q g}\left(\frac{x}{y}\right) \frac{x}{y} G\left(\frac{x}{y}, \mu^{2}\right)\right] .
\end{aligned}
$$

Here, $P_{q q}\left(\frac{x}{y}\right)$ and $P_{q g}\left(\frac{x}{y}\right)$ are known as DGLAP splitting functions. They are differential equations which describe to leading-logarithmic accuracy the change in the parton distribution functions when changing $\mu^{2}$, and are an important example of what one calls evolution equations in quantum field theory. Solving them amounts to the resummation of all the leading-order collinear QCD corrections to deep inelastic scattering processes. Equivalently, the DGLAP equations can be regarded as renormalization-group equations, which renormalize the parton densities with respect to the scale $\mu^{2}$. The DGLAP equation allows us to explain the phenomenon of the scaling violation of the proton structure function.

The dependence of the DA on the factorization scale $\mu_{F}^{2}$ is governed by the Efremov-Radyushkin-Brodsky-Lepage (ERBL) evolution equation [1-3] which is defined the following form:

$$
\frac{\partial \Phi\left(x, \mu_{F}^{2}\right)}{\partial \ln \mu_{F}^{2}}=\int d y V\left(x, y, \alpha_{s}\left(\mu_{F}^{2}\right)\right) \Phi\left(y, \mu_{F}^{2}\right) .
$$

The evolution kernel $V\left(x, y, \alpha_{s}\left(\mu_{F}^{2}\right)\right)$ is calculable in perturbation theory

$$
V\left(x, y, \alpha_{s}\left(\mu_{F}^{2}\right)\right)=\frac{\alpha_{s}\left(\mu_{F}^{2}\right)}{\pi} V_{1}(x, y)+\left(\frac{\alpha_{s}\left(\mu_{F}^{2}\right)}{\pi}\right)^{2} V_{2}(x, y) .
$$

The one-loop evolution kernel $V_{0}$ was introduced in Refs. [1,7], an analogous expression for $V_{2}$ at the two-loop level was derived in Refs. [17-20]. It should be noted that the HT refers to contributions suppressed by powers of large momentum with respect to the leading twist. The leading twist (LT) is a standard process of the pQCD within the collinear factorization where hadrons are produced through fragmentation processes. However, HT processes are taken usually as direct hadron production, where the hadron is produced directly in the hard subprocess rather than by quark/gluon fragmentation. Higher-twist dynamics at the hadron production in hadron-hadron collisions is widely studied in Ref. [21].

In Refs. [22,23], it is showed that hard-scattering factorization is disrupted in the production of high- $p_{T}$ hadrons in the case of the hadrons being back-to-back by using $k_{T}$ factorization. It is worthy noted that perturbative QCD factorization formulas are modified at leading twist by initial and final state corrections. The explicit counterexample was provided for the single-spin asymmetry with one beam transversely polarized as well. The calculation and analysis the contribution of the HT effects to cross section on the dependence of the pion DA in inclusive pion pair production at $p \bar{p}$ collision within the frozen coupling constant (FCC) approach are important and interesting research problems. Therefore, HT effects in QCD have been predicted and computed in the last 40 years by many researchers for various phenomena [24-39]. Meson pair production in photon-photon, nucleon-nucleon, and proton-antiproton collisions have been studied from high to low energies during the last few years, applying different approaches such as HT mechanism, central exclusive production mechanism, effective meson theory, and standard pQCD [40-47].

Precision experimental studies of meson pair production in proton-antiproton collisions at low energies are proposed in the experiment named PANDA [48]. The PANDA scientific program uses $1.5-15 \mathrm{GeV}$ energy range for interactions between protons and antiprotons where this energy lies near the pion production threshold. This program includes several measurements and it addresses fundamental questions of QCD by obtaining the detailed analyses of all possible mechanisms of meson pair production [49]. In this study, we examine the contribution of the HT effects to inclusive charged pion pair production at proton-antiproton collisions by using different pion DAs obtained within holographic and perturbative QCD which can be helpful for an explanation of the PANDA experiment. We have also given theoretical predictions of the inclusive charged pion pair production in $p \bar{p}$ collisions by accounting for the leading order diagrams in partonic cross sections.

The physical information of the inclusive pion pair production can be obtained efficiently in the PQCD and it is, hence, possible to compare directly with the experimental data. The corresponding hard-scattering subprocesses occur via three different mechanisms. The first one is the direct production of charged pion pairs which are produced directly at the hard-scattering subprocess (see Fig. 1). The second one is the semidirect production of charged pion pairs in which one pion is produced from jet fragmentation (see Fig. 2). Finally, the last one is the double jet production and fragmentation where both pions are produced from fragmentation of the final quarks or gluons. The first two mechanisms are HT contributions and the last is the LT contribution. Therefore, we must systematically compare these different mechanisms. We use 


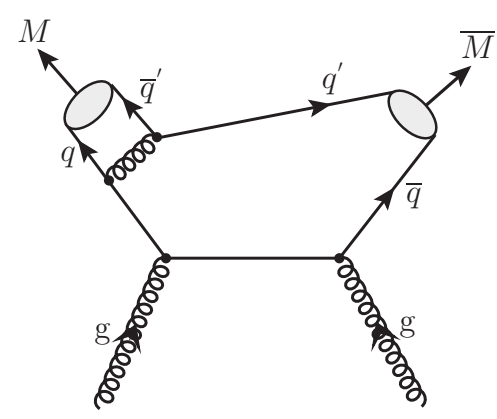

(a)

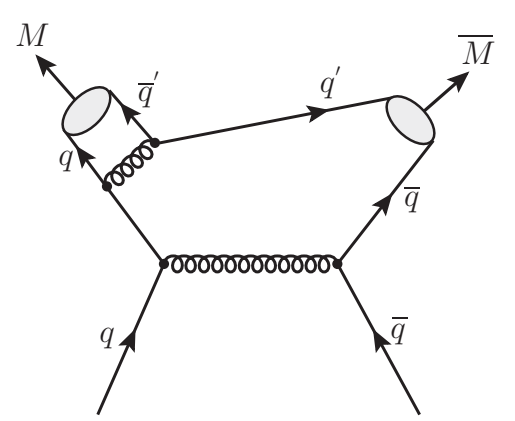

(d)

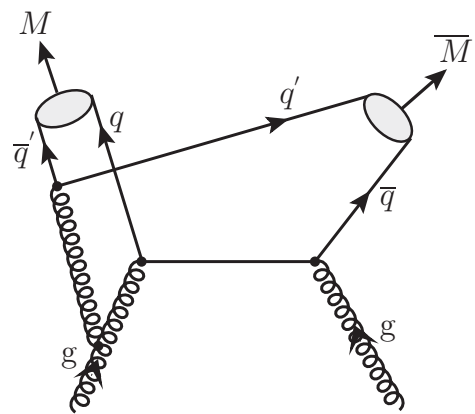

(b)

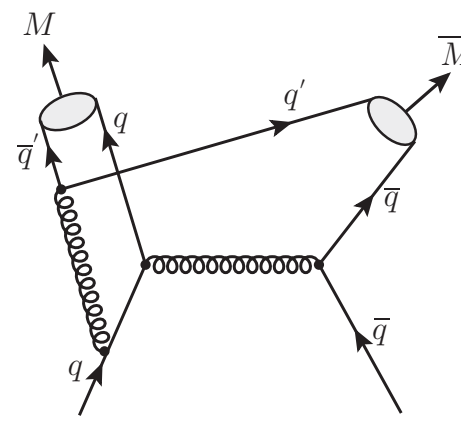

(e)

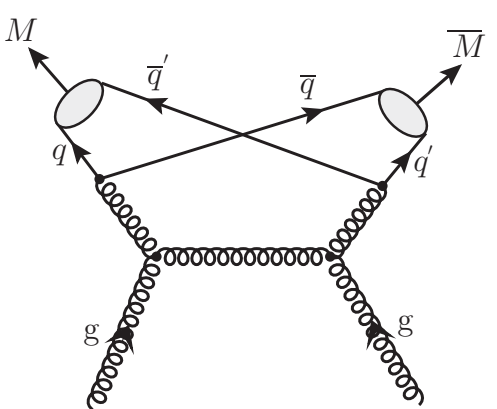

(c)

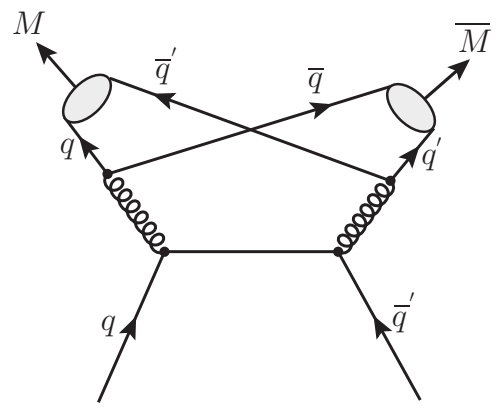

(f)

FIG. 1. QCD Feynman diagrams of the partonic process $g g \rightarrow M \bar{M}$ and $q \bar{q} \rightarrow M \bar{M}$ for direct meson pair production at leading order.

the frozen coupling constant (FCC) approach during numerical evaluation in all calculations. In order to obtain an accurate value of the ratio (HT/LT), we need to use the fact that prompt pions appear "nonaccompanied" by any other hadron, while this is not valid for the general case in which particles are resulting from the jet fragmentation. That criterion of "nonaccompaniment" into the general formalizing a momentum cutoff parameter $\Delta p$ is considered in calculation [50].

The rest of the paper is organized as follows. In Sec. II, a brief review for the formalism used for the calculation of the

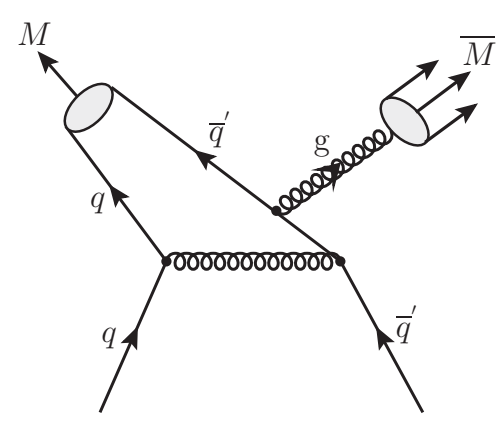

(a)

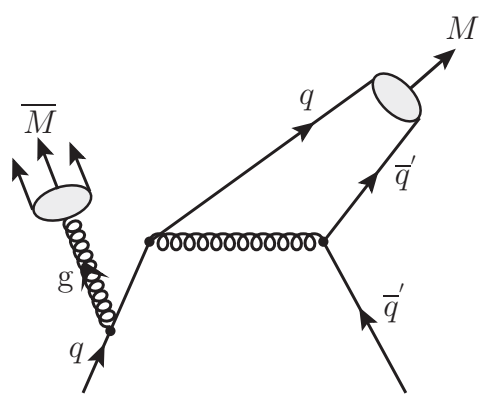

(d)

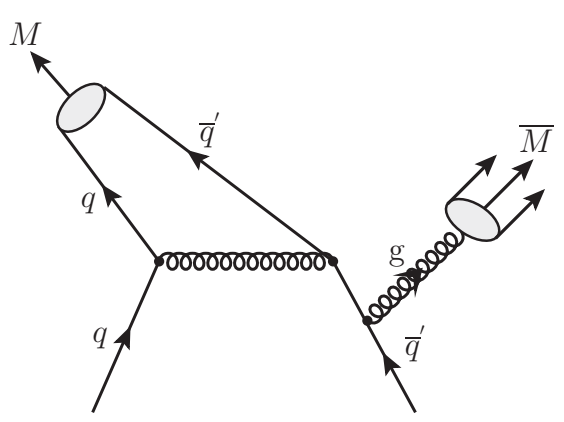

(b)

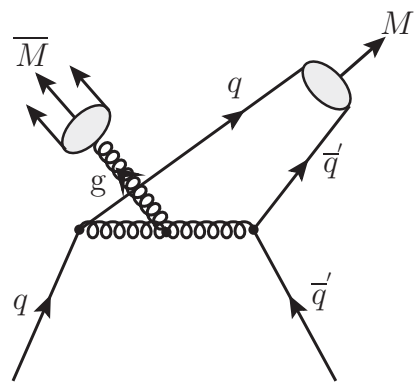

(e)

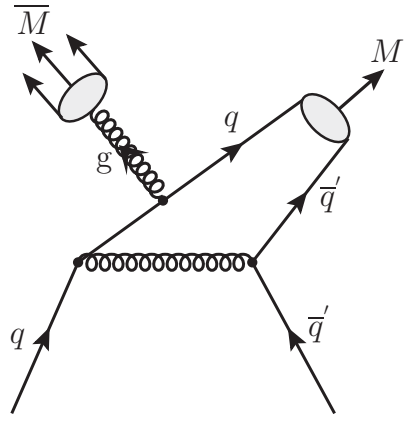

(c)

FIG. 2. QCD Feynman diagrams of the partonic process $q \bar{q} \rightarrow M \bar{M}$ for semidirect meson pair production at leading level. 
HT contribution to cross section and some formulas for the HT cross section of the process $p \bar{p} \rightarrow \pi^{+} \pi^{-} X$ is given. In Sec. III, some formulas for LT cross sections for pion pairs production are provided. In Sec. IV, we present a comparison of the HT charged pion pair production $p \bar{p} \rightarrow \pi^{+} \pi^{-}$ cross section with elastic $\pi^{ \pm} p \rightarrow \pi^{ \pm} p$ cross section, and the numerical results for the cross section and the discussion of the dependence on the cross section on the pion DA are provided in Sec. V. Finally, the concluding remarks are stated in Sec. VI.

\section{HIGHER-TWIST CONTRIBUTION TO INCLUSIVE DIRECT PION PAIR PRODUCTION CROSS SECTION}

The inclusive production of charged pion pair with large transverse momenta $\left(p_{T}>1 \mathrm{GeV} / c\right)$ in opposite hemispheres, essentially back-to-back in the center-of-mass system of the incoming hadrons is considered in this study. This mechanism had been already analyzed in Ref. [50] for the case of the two particle back-to-back cross section reflecting the $p_{T}$ dependence of the hard scattering subprocesses undisturbed by the internal momenta of the constituents. There are many other studies in the literature about physical properties of FCC [51-64]. In numerically calculating the HT cross section (within FCC approach for the square of the transfer momentum of the hard gluon) and LT cross section we can use the following values as

$$
Q^{2}= \begin{cases}\frac{1}{2} p_{T}^{2} & \text { for direct HT contribution } \\ \frac{1}{2} \frac{p_{T}^{2}}{\sqrt{z}} & \text { for semidirect HT contribution } \\ \frac{1}{2} \frac{p_{T}^{2}}{\sqrt{z z^{\prime}}} & \text { for LT contribution. }\end{cases}
$$

Using the fact that prompt pions are nonaccompanied by any other hadron, the ratio contributions of HT and LT can be calculated accurately. However, this is not valid for the general case in which particles are occurring from the jets fragmentation. This criterion can be incorporated into the general formulas via a momentum cut-off parameter $\Delta p$ [29]. The details of analytical calculations on HT and LT contributions will be given in the following subsections. The leading order HT Feynman subdiagrams for the inclusive direct pion pair production in the proton-antiproton collision $p \bar{p} \rightarrow \pi^{+} \pi^{-} X$ are taken as $g g \rightarrow \pi^{+} \pi^{-}$and $q \bar{q} \rightarrow \pi^{+} \pi^{-}$(where $q$ is either $u$ or $d$ quarks) which contribute to the main process (see
Fig. 1). Semidirect pion pair productions in the same process are shown in Fig. 2. The amplitude for this subprocess can be obtained by using the Brodsky-Lepage formula [7]

$$
\begin{aligned}
M(\hat{s}, \hat{t})= & \int_{0}^{1} d x_{1} \int_{0}^{1} d x_{2} \delta\left(1-x_{1}-x_{2}\right) \Phi_{M}\left(x_{1}, x_{2}, Q^{2}\right) \\
& \times T_{H}\left(x_{1}, x_{2} ; Q^{2}, \mu_{R}^{2}, \mu_{F}^{2}\right)
\end{aligned}
$$

where $T_{H}$ is the sum of the graphs contributing to the hard-scattering part of the subprocess. At the leading order of pQCD calculations, the hard scattering amplitude $T_{H}\left(x_{1}, x_{2} ; Q^{2}, \mu_{R}^{2}, \mu_{F}^{2}\right)$ does not depend on the factorization scale $\mu_{F}^{2}$, but strongly depends on $\mu_{R}^{2}$. However, the scales $\mu_{F}^{2}$ and $\mu_{R}^{2}$ are independent of each other.

In principle, all measurable quantities in QCD should be invariant under any choice of renormalization scale and scheme. It is clear that the use of different scales and schemes may lead to different theoretical predictions. Therefore, the constructive mathematical tool for defining QCD is a choice of the renormalization scale which makes scheme independent results at all fixed order in running coupling constant $\alpha_{s}$. For direct pion pair production, the subprocesses are taken as $g g \rightarrow \pi^{+} \pi^{-}, u \bar{u} \rightarrow \pi^{+} \pi^{-}$, and $d \bar{d} \rightarrow \pi^{+} \pi^{-}$. However, for the semidirect pion pair production the subprocesses are $q \bar{q} \rightarrow \pi g, q g \rightarrow \pi q^{\prime}$, and $\bar{q} g \rightarrow \pi \bar{q}^{\prime}$. In the processes $q \bar{q} \rightarrow$ $\pi g$, the final gluon is $q g \rightarrow \pi q^{\prime}$, the final quark is $\bar{q} g \rightarrow \pi \bar{q}^{\prime}$, and the final antiquark is taken as a fragmentation of the pion. Here, $q, \bar{q}$, and $g$ are the constituent of the initial target proton and antiproton. It should be noted that each $q \bar{q}$ pair is collinear and has the appropriate color, spin, and flavor content projected out to form the parent pion. The production of the pair of pions or jets in the large transverse momentum is available at the high energy, especially at the CERN Large Hadron Collider. In the direct pion pair production case, the hadronic pion is the final product of the hard-scattering processes. But in the final state of the semidirect pion pair production, one of the hadronic gluon or jets are fragmented to a pion. Dynamical properties of the jet are close to the parent parton which are carried by one of part of the four-momentum of the parent parton. In order to explain parton level kinematics, we use the pion pair production process considered in Ref. [65].

The parton-level differential cross sections for the direct pion pair production are obtained as

$$
\begin{aligned}
\frac{d \sigma}{d \cos \theta}\left(g g \rightarrow \pi^{+} \pi^{-}\right)= & \frac{256 \pi^{3} \alpha_{s}^{4} f_{\pi}^{4}}{23328}\left[\int_{0}^{1} \frac{\Phi_{\pi}\left(x, Q^{2}\right) d x}{x(1-x)}\right]^{2}\left[\int_{0}^{1} d x \int_{0}^{1} d y \frac{\Phi_{\pi}\left(x, Q^{2}\right) \Phi_{\pi}\left(y, Q^{2}\right)}{x(1-x) y(1-y)} \frac{x(1-x)+y(1-y)}{x y+(1-x)(1-y)}\right]^{2}, \\
\frac{d \sigma}{d \cos \theta}\left(q \bar{q} \rightarrow \pi^{+} \pi^{-}\right)= & \frac{256 \pi^{3} \alpha_{s}^{4} f_{\pi}^{4}}{139968}\left[\int_{0}^{1} \frac{\Phi_{\pi}\left(x, Q^{2}\right) d x}{x(1-x)}\right]^{2}\left[\int_{0}^{1} d x \int_{0}^{1} d y \frac{\Phi_{\pi}\left(x, Q^{2}\right) \Phi_{\pi}\left(y, Q^{2}\right)}{x(1-x) y(1-y)} \frac{x(1-x)+y(1-y)}{x y+(1-x)(1-y)}\right]^{2} \\
& \times\left[7-16 x y-\frac{1}{x y+(1-x)(1-y)}\left[2 x(1-2 y(x+y))-4 x^{2}+4 x y\right]\right] .
\end{aligned}
$$

Similarly, for the semidirect pion pair production case which corresponds to the Feynman diagrams in Fig. 2, the hard collisions subprocesses are taken in three different ways as 
(1) $q \bar{q}^{\prime} \rightarrow \pi^{+}\left(\pi^{-}\right) g$, where the gluon is fragmented to a pion $\left(g \rightarrow \pi^{-}\left(\pi^{+}\right)\right)$,

(2) $q g \rightarrow \pi^{ \pm} q^{\prime}$, where the quark is fragmented to a pion $\left(q^{\prime} \rightarrow \pi^{\mp}\right)$

(3) $\bar{q} g \rightarrow \pi^{ \pm} \bar{q}^{\prime},\left(\bar{q}^{\prime} \rightarrow \pi^{\mp}\right)$, where the antiquark is fragmented to a pion.

The corresponding differential cross sections of the subprocesses are defined for these cases as

$$
\begin{aligned}
& \frac{d \sigma}{d \cos \theta}\left(q \overline{q^{\prime}} \rightarrow \pi^{ \pm} g\right)=\frac{128 \pi^{2} \alpha_{s}^{3} f_{\pi}^{2}}{729 \hat{s}^{2}}\left[\int_{0}^{1} \frac{\Phi_{\pi}\left(x, Q^{2}\right) d x}{x(1-x)}\right]^{2}, \\
& \frac{d \sigma}{d \cos \theta}\left(q g \rightarrow \pi^{ \pm} q^{\prime}\right)=\frac{80 \pi^{2} \alpha_{s}^{3} f_{\pi}^{2}}{3888 \hat{s}^{2}}\left[\int_{0}^{1} \frac{\Phi_{\pi}\left(x, Q^{2}\right) d x}{x(1-x)}\right]^{2}, \\
& \frac{d \sigma}{d \cos \theta}\left(\bar{q} g \rightarrow \pi^{ \pm} \bar{q}^{\prime}\right)=\frac{80 \pi^{2} \alpha_{s}^{3} f_{\pi}^{2}}{3888 \hat{s}^{2}}\left[\int_{0}^{1} \frac{\Phi_{\pi}\left(x, Q^{2}\right) d x}{x(1-x)}\right]^{2},
\end{aligned}
$$

respectively. The main goals of this study are the calculation and also, if possible, extraction of the contributions HT effects to the cross section by the FCC approach using different pion DAs. For the calculation of the cross section, we need to apply the factorization formula which was predicted by Gunion and Petersson $[66,67]$. In this approach a differential cross section of the process $p \bar{p} \rightarrow \pi^{+} \pi^{-} X$ is defined as

$$
\begin{aligned}
\Sigma_{\pi^{+} \pi^{-}}= & E_{C} E_{D} \frac{d \sigma}{d^{3} p_{C} d^{3} p_{D}} \\
= & \frac{1}{\pi^{2} s\left\langle q_{T}^{2}\right\rangle} \int_{z_{\min }}^{1} \frac{d z}{z^{2}} \int_{z_{\min }}^{1} \frac{d z^{\prime}}{z^{\prime 2}} F\left(z, z^{\prime}\right) \\
& \times G_{q_{1} / p_{1}}\left(x_{1}, Q^{2}\right) G_{q_{2} / p_{2}}\left(x_{2}, Q^{2}\right) \\
& \times \frac{d \sigma}{d \cos \theta}\left(q \bar{q}(g g) \rightarrow \pi^{+} \pi^{-}\right) \\
& \times D_{M / C}\left(z, Q^{2}\right) D_{\bar{M} / D}\left(z^{\prime}, Q^{2}\right),
\end{aligned}
$$

where $s$ is the center-of-mass energy squared of main process, $\left\langle q_{T}^{2}\right\rangle$ is the mean square of the intrinsic momentum of either initial parton $q_{1}, q_{2}, G_{q_{1} / p_{1}}$ and $G_{q_{2} / p_{2}}$ are the universal PDFs for the partons $q_{1}, q_{2}$ in the proton and antiproton $p_{1}, p_{2}$, respectively. They depend on the longitudinal momentum fractions of the two partons in the case when final jets are fragmenting to pion pair $x_{1}=x_{2}=2 p_{T} / \sqrt{z z^{\prime} s}$ and on the scale parameter $Q^{2}$ of the central collision process. $d \sigma / d \cos \theta$ is the differential cross section of the process and $\theta$ is the scattering angle. In the main process, both pions are emitted at $90^{\circ}$ in the center-of-mass frame. For the dependence of the symmetric pair production cross section $E_{C} E_{D} \frac{d \sigma}{d^{3} p_{C} d^{3} p_{D}}$ at $90^{\circ}$ of the transverse momentum, we take into account $p_{T}=p_{T_{C}}=-p_{T_{D}}, y_{C}=y_{D}=0, \varphi_{C}=0$, and $\varphi_{D}=\pi$.

The longitudinal momentum fractions of partons are defined in this form

$$
\begin{aligned}
& x_{1}=-\frac{1}{2}\left(x_{T_{1}} e^{y_{1}}+x_{T_{2}} e^{y_{2}}\right), \\
& x_{2}=-\frac{1}{2}\left(x_{T_{1}} e^{-y_{1}}+x_{T_{2}} e^{-y_{2}}\right),
\end{aligned}
$$

in which $y_{1}, y_{2}$ are the rapidities of the final particles.

For the calculation of the HT cross sections in the case of direct pion pair production, we assume in Eq. (2.8) that $M=\pi^{+}, C=\pi^{+}$and $\bar{M}=\pi^{-}, D=\pi^{-}$. Therefore instead of fragmentation functions (FFs) $D_{M / C}\left(z, Q^{2}\right)$ and $D_{\bar{M} / D}\left(z^{\prime}, Q^{2}\right)$, we make the substitutions $D_{\pi^{+} / \pi^{+}}\left(z, Q^{2}\right)=$ $\delta(1-z)$ and $D_{\pi^{-} / \pi^{-}}\left(z^{\prime}, Q^{2}\right)=\delta\left(1-z^{\prime}\right)$. But, for the HT cross section in the semidirect pion pair production case, we take $M=\pi^{+}, C=\pi^{+}$, then we make the substitutions $D_{\pi^{+} / \pi^{+}}\left(z, Q^{2}\right)=\delta(1-z)$. In the numerical calculations, the function fragmentation of the gluon and quark [68] into a pion have been used. The function $F\left(z, z^{\prime}\right)$ called the correlation function is defined as

$$
F\left(z, z^{\prime}\right)=\frac{z+z^{\prime}}{2 \sqrt{z z^{\prime}}} \exp \left[\frac{-\left(z-z^{\prime}\right)^{2} p_{T}^{2}}{2 z^{2} z^{\prime 2}\left\langle q_{T}^{2}\right\rangle}\right] .
$$

In the LT subprocess, the pion is indirectly emitted from the quark with fractional momentum $z$. The minimum value of the momentum fraction of the final parton $z_{\min }$ is defined in this form:

$$
z_{\min }=\frac{p_{T}}{p_{T}+\triangle p} .
$$

Here, $\triangle p$ is a momentum cut-off parameter which describes the experimental upper limit for nondetection of one or more particles accompanying either pion detected. It is assumed that whenever this limit is exceeded, the corresponding event will be rejected.

\section{LEADING-TWIST CONTRIBUTIONS TO INCLUSIVE CHARGED PION PAIR PRODUCTION CROSS SECTION}

It is an important task to compare the HT corrections with LT contributions and to extract the HT corrections to the pion pair production cross section.

For the LT cross section for the production of pion pairs, we take the next subprocesses in which the final particles are fragmented to pion pairs as $q \bar{q} \rightarrow g g\left(g \rightarrow \pi^{+}, g \rightarrow \pi^{-}\right)$, $g g \rightarrow q \bar{q}\left(q \rightarrow \pi^{+}, \bar{q} \rightarrow \pi^{-}\right), q g \rightarrow q g\left(q \rightarrow \pi^{+}, g \rightarrow \pi^{-}\right)$, $g g \rightarrow g g\left(g \rightarrow \pi^{+}, g \rightarrow \pi^{-}\right)$, and $q \bar{q} \rightarrow q \bar{q}\left(q \rightarrow \pi^{+}, \bar{q} \rightarrow\right.$ $\left.\pi^{-}\right)$.

The corresponding differential cross section of the LT subprocesses are written as [65]

$$
\begin{aligned}
\frac{d \sigma}{d \cos \theta}\left(q_{1} q_{2} \rightarrow q_{1} q_{2}\right) & =\frac{2 \pi \alpha_{s}^{2}}{9 \hat{s}}\left(\frac{u^{2}+s^{2}}{t^{2}}\right), \\
\frac{d \sigma}{d \cos \theta}\left(q_{1} \bar{q}_{2} \rightarrow q_{1} \bar{q}_{2}\right) & =\frac{2 \pi \alpha_{s}^{2}}{9 \hat{s}}\left(\frac{u^{2}+s^{2}}{t^{2}}\right),
\end{aligned}
$$




$$
\begin{aligned}
\frac{d \sigma}{d \cos \theta}\left(q_{1} q_{1} \rightarrow q_{1} q_{1}\right) & =\frac{\pi \alpha_{s}^{2}}{2 \hat{s}}\left(\frac{4}{9}\left(\frac{u^{2}+s^{2}}{t^{2}}+\frac{s^{2}+t^{2}}{u^{2}}\right)-\frac{8}{27} \frac{s^{2}}{u t}\right) \\
\frac{d \sigma}{d \cos \theta}\left(q_{1} \bar{q}_{1} \rightarrow q_{2} \bar{q}_{2}\right) & =\frac{2 \pi \alpha_{s}^{2}}{9 \hat{s}}\left(\frac{u^{2}+t^{2}}{s^{2}}\right) \\
\frac{d \sigma}{d \cos \theta}\left(q_{1} \bar{q}_{1} \rightarrow q_{1} \bar{q}_{1}\right) & =\frac{\pi \alpha_{s}^{2}}{2 \hat{s}}\left(\frac{4}{9}\left(\frac{u^{2}+s^{2}}{t^{2}}+\frac{u^{2}+t^{2}}{s^{2}}\right)-\frac{8}{27} \frac{u^{2}}{s t}\right), \\
\frac{d \sigma}{d \cos \theta}(q \bar{q} \rightarrow g g) & =\frac{\pi \alpha_{s}^{2}}{2 \hat{s}}\left(\frac{32}{27} \frac{u^{2}+t^{2}}{u t}-\frac{8}{3} \frac{u^{2}+t^{2}}{s^{2}}\right) \\
\frac{d \sigma}{d \cos \theta}(g g \rightarrow q \bar{q}) & =\frac{\pi \alpha_{s}^{2}}{2 \hat{s}}\left(\frac{1}{6} \frac{u^{2}+t^{2}}{u t}-\frac{3}{8} \frac{u^{2}+t^{2}}{s^{2}}\right) \\
\frac{d \sigma}{d \cos \theta}(q g \rightarrow q g) & =\frac{\pi \alpha_{s}^{2}}{2 \hat{s}}\left(-\frac{4}{9} \frac{u^{2}+s^{2}}{u s}+\frac{u^{2}+s^{2}}{t^{2}}\right) \\
\frac{d \sigma}{d \cos \theta}(g g \rightarrow g g) & =\frac{\pi \alpha_{s}^{2}}{\hat{s}} \frac{9}{4}\left(3-\frac{u t}{s^{2}}-\frac{u s}{t^{2}}-\frac{s t}{u^{2}}\right) \\
\frac{d \sigma}{d \cos \theta}\left(q_{1} q_{1} \rightarrow q_{2} q_{2}\right) & =\frac{2 \pi \alpha_{s}^{2}}{9 \hat{s}}\left(\frac{u^{2}+t^{2}}{s^{2}}\right)
\end{aligned}
$$

where subscripts 1 and 2 denote distinct flavors. The initial and final state colors and spins have been averaged and summed, respectively. Over the last few years, a great deal of progress has been made in the investigation of the properties of hadronic wave functions. The notion of distribution amplitudes refers to momentum fraction distributions of partons in the meson, in particular, the Fock state with a fixed number of components. For the minimal number of constituents, the distribution amplitude $\Phi$ is related to the Bethe-Salpeter wave function $\Phi_{B S}$ by

$$
\Phi(x) \sim \int^{\left|k_{\perp}\right|<\mu} d^{2} k_{\perp} \Phi_{B S}\left(x, k_{\perp}\right) .
$$

The standard approach to distribution amplitudes, which is due to Brodsky and Lepage [69], considers the hadron's parton decomposition in the infinite momentum frame. A conceptually different, but mathematically equivalent formalism is the light-cone quantization [70]. The meson distribution amplitudes play a key role in the hard-scattering QCD processes because they encapsulate the essential nonperturbative features of the meson's internal structure in terms of the parton's longitudinal momentum fractions $x_{i}$. Meson DAs have been extensively studied by using QCD sum rules. The original suggestion by Chernyak and Zhitnitsky of a "doublehumped" wave function of the pion at a low scale, far from the asymptotic form, was based on an extraction of the first few moments from a standard QCD sum rule approach [71], in the Bakulev-Mikhailov-Stefanis (BMS) DA two nontrivial Gegenbauer coefficients $a_{2}$ and $a_{4}$ have been extracted from the CLEO data on the $\gamma \gamma^{\star} \rightarrow \pi^{0}$ transition form factor in which the authors have used the QCD light-cone sum rules approach and have included in their analysis the next to leading order perturbative and twist-four corrections. Thus, in our numerical calculations, we used several choices, such as the asymptotic DAs predicted by pQCD evaluation, light-cone formalism, the light-front quark model [1], the Vega-SchmidtBranz-Gutsche-Lyubovitskij (VSBGL) DA [72], holographic meson DAs obtained in the context of AdS/CFT ideas [73,74] are studied considering two kinds of holographic soft-wall models, the Chernyak-Zhitnitsky(CZ) [71], and the BMS [75]:

$$
\begin{aligned}
\Phi_{\mathrm{asy}}(x) & =\sqrt{3} f_{\pi} x(1-x), \\
\Phi_{\mathrm{VSBGL}}^{\mathrm{hol}}(x) & =\frac{A_{1} k_{1}}{2 \pi} \sqrt{x(1-x)} \exp \left(-\frac{m^{2}}{2 k_{1}^{2} x(1-x)}\right), \\
\Phi^{\mathrm{hol}}(x)= & \frac{4}{\sqrt{3} \pi} f_{\pi} \sqrt{x(1-x)}, \\
\Phi_{\mathrm{CZ}}\left(x, \mu_{0}^{2}\right)= & \Phi_{\mathrm{asy}}(x)\left[C_{0}^{3 / 2}(2 x-1)+\frac{2}{3} C_{2}^{3 / 2}(2 x-1)\right], \\
\Phi_{\mathrm{BMS}}\left(x, \mu_{0}^{2}\right)= & \Phi_{\text {asy }}(x)\left[C_{0}^{3 / 2}(2 x-1)+0.20 C_{2}^{3 / 2}(2 x-1)\right. \\
& \left.-0.14 C_{4}^{3 / 2}(2 x-1)\right] .
\end{aligned}
$$

The pion DA can be expanded over the eigenfunctions of the one-loop ERBL equation

$$
\Phi_{\pi}\left(x, Q^{2}\right)=\Phi_{\text {asy }}(x)\left[1+\sum_{n=2,4 . .}^{\infty} a_{n}\left(Q^{2}\right) C_{n}^{3 / 2}(2 x-1)\right] .
$$

The evolution of the DA on the factorization scale $Q^{2}$ is governed by the functions $a_{n}\left(Q^{2}\right)$ as

$$
\begin{aligned}
a_{n}\left(Q^{2}\right) & =a_{n}\left(\mu_{0}^{2}\right)\left[\frac{\alpha_{s}\left(Q^{2}\right)}{\alpha_{s}\left(\mu_{0}^{2}\right)}\right]^{\gamma_{n} / \beta_{0}}, \\
\frac{\gamma_{2}}{\beta_{0}} & =\frac{50}{81}, \quad \frac{\gamma_{4}}{\beta_{0}}=\frac{364}{405}, \quad n_{f}=3 .
\end{aligned}
$$


In Eq. (3.18), $\gamma_{n}$ 's are anomalous dimensions defined by the expression

$$
\gamma_{n}=C_{F}\left[1-\frac{2}{(n+1)(n+2)}+4 \sum_{j=2}^{n+1} \frac{1}{j}\right] .
$$

The Gegenbauer moments $a_{n}$ can be determined by using the Gegenbauer polynomials orthogonality condition

$$
\int_{-1}^{1}\left(1-\zeta^{2}\right) C_{n}^{3 / 2}(\zeta) C_{n^{\prime}}^{3 / 2}(\zeta) d \zeta=\frac{\Gamma(n+3) \delta_{n n^{\prime}}}{n !(n+3 / 2)}
$$

The Gegenbauer moments $a_{n}$ are very practical in studying the DAs because they form the shape of the corresponding hadron wave function. Hereby, it can be possible to derive from theoretical models or extract from the experimental data. Besides, these moments reveal how much the DAs deviate from the asymptotic one. The strong coupling constant $\alpha_{s}\left(Q^{2}\right)$ at the one-loop approximation is given as

$$
\alpha_{s}\left(Q^{2}\right)=\frac{4 \pi}{\beta_{0} \ln \left(\frac{Q^{2}}{\Lambda^{2}}\right)}
$$

where $\Lambda$ is the QCD scale parameter, $\beta_{0}$ is the QCD beta function one-loop coefficients. It should be noted that the choice of renormalization scale in $\alpha_{s}\left(Q^{2}\right)$ is one of the main problems in QCD. In the numerical calculations, the hard gluon square momentum was used from Eq. (2.1). Notice that the pion DAs presented in Eqs. (3.12)-(3.16) constructed from theory and experiment strongly depend on the applied methods. However, the correct pion wave function is still an open problem in QCD.

\section{COMPARISON HIGHER-TWIST PION PAIR PRODUCTION CROSS SECTION $p \bar{p} \rightarrow \pi^{+} \pi^{-}$WITH ELASTIC $\pi^{ \pm} p \rightarrow \pi^{ \pm} p$ CROSS SECTION}

It would be important and interesting to compare the proton-antiproton annihilation process $p \bar{p} \rightarrow \pi^{+} \pi^{-}$with the elastic backward scattering $\pi^{ \pm} p \rightarrow \pi^{ \pm} p$ process by fixing $u$ and switching $s$ and $t$. In order to compare matrix elements at given values $s, t$, or $u$, spin and phase-space factors have to be taken into account more specifically. We compare the differential cross section for the annihilation process $\frac{d \sigma}{d t}(p \bar{p} \rightarrow$ $\pi^{+} \pi^{-}$) with the corresponding elastic backward cross section $\frac{d \sigma}{d t}\left(\pi^{ \pm} p \rightarrow \pi^{ \pm} p\right)$ using the suitable spin and phase-space factors. So,

$$
\begin{aligned}
& \frac{d \sigma}{d t}\left(p \bar{p} \rightarrow \pi^{+} \pi^{-}\right) \\
& \quad=\frac{\left(2 s_{\pi}+1\right)\left(2 s_{p}+1\right)}{\left(2 s_{\bar{p}}+1\right)\left(2 s_{p}+1\right)}\left(\frac{k_{\pi p}}{p_{\bar{p} p}}\right)^{2} \frac{d \sigma}{d t}\left(\pi^{ \pm} p \rightarrow \pi^{ \pm} p\right),
\end{aligned}
$$

where $s_{\pi}$ and $s_{p}\left(s_{\bar{p}}\right)$ are the spins of the pion and proton(antiproton), $k_{\pi p}$ and $p_{\bar{p} p}$ are the center of mass momenta, evaluated at the same center-of-mass energy. If the hadrons are produced at $90^{\circ}$ with rapidities $y_{C}=y_{D}=0$, the hard scattering cross section $d \sigma / d \hat{t}$ is probed at angles around $90^{\circ}$, where $\hat{t}=\hat{u}=-\hat{s} / 2$. The comparison is relevant only at the center of mass energies and therefore the elastic backward cross sections are scaled by using an $s^{-2}$ dependence for $\pi p$. The result of the comparison are present in Figs. 13 and 14.

\section{NUMERICAL RESULTS AND DISCUSSION}

Let us now discuss in detail the numerical predictions of the HT and LT cross sections of the pion pair production process $p \bar{p} \rightarrow \pi^{+} \pi^{-} X$ at the PANDA energies taking into account the full leading-order contributions from quarkantiquark annihilation and gluon-gluon fusion. We denote the HT cross section by $\Sigma_{\pi^{+} \pi^{-}}^{\mathrm{HT}}$, the LT cross section by $\Sigma_{\pi^{+} \pi^{-}}^{\mathrm{LT}}$, and the sum of HT and LT by $\Sigma_{\pi^{+}+\pi^{-}}^{\mathrm{HT}+\mathrm{LT}}$. For the quark and gluon distribution functions inside the proton and antiproton, the MSTW2008 PDFs [76] and the quark and gluon fragmentation functions [68] are used. Also, the following abbreviations are defined: asy is $\Phi_{\text {asy }}(x)$, hol is $\Phi^{\text {hol }}(x)$, VSBGL is $\Phi_{\mathrm{VSBGL}}^{\text {hol }}(x), \mathrm{CZ}$ is $\Phi_{\mathrm{CZ}}\left(x, Q^{2}\right)$, and BMS is $\Phi_{\mathrm{BMS}}\left(x, Q^{2}\right)$. The results are given for $\sqrt{s}=15$ and $20 \mathrm{GeV}$ on the transverse momentum $p_{T}$ ranging from $1 \mathrm{GeV} / c$ to $7 \mathrm{GeV} / c$ which are also valid for the PANDA experiment. Obtained results are visualized through in Figs. 3-16.

First, we compare the HT and LT cross sections obtained within holographic QCD and pQCD. In Figs. 3 and 4, we show the HT cross section $\Sigma_{\pi^{+} \pi^{-}}^{\mathrm{HT}}$ and the sum of HT and LT cross sections $\Sigma_{\pi^{+}+\pi^{-}}^{\mathrm{HT}+\mathrm{LT}}$ which are calculated in the context of the FCC approach as a function of the pion pair transverse momentum $p_{T}$ for the pion DAs for Eqs. (3.12)-(3.16) and for $y=0$. It is also seen that the $\Sigma_{\pi^{+} \pi^{-}}^{\mathrm{HT}}$ and $\Sigma_{\pi^{+}+\pi^{-}}^{\mathrm{HT}+\mathrm{LT}}$ cross sections are monotonically decreasing with an increase in the transverse momentum of the pion pair. It is worth to mention that at the c.m. energy $\sqrt{s}=15 \mathrm{GeV}$ the maximum value of the frozen cross section of the process $p \bar{p} \rightarrow \pi^{+} \pi^{-} X$ for the $\Phi_{\mathrm{CZ}}\left(x, Q^{2}\right)$ decreases from the interval $2.10992 \times 10^{-3} \mathrm{mb} / \mathrm{GeV}^{4}$ to $1.32239 \times$ $10^{-33} \mathrm{mb} / \mathrm{GeV}^{4}$, but the $\Sigma_{\pi^{+} \pi^{-}}^{\mathrm{HT}+\mathrm{LT}}$ cross sections for the same

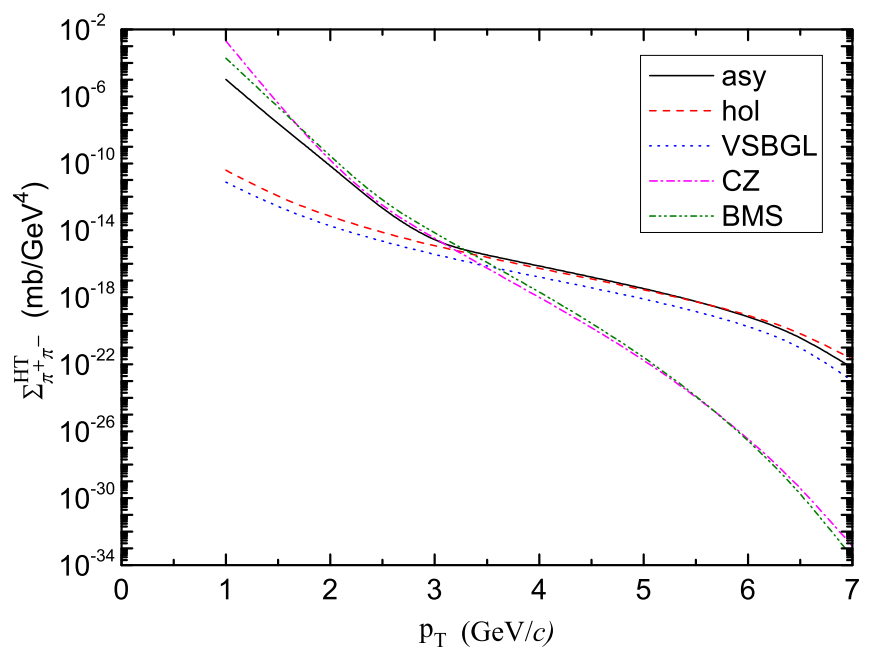

FIG. 3. HT contribution to charged pion pair production $p \bar{p} \rightarrow$ $\pi^{+} \pi^{-} X$ cross section $\Sigma_{\pi^{+} \pi^{-}}^{\mathrm{HT}}$ as a function of the transverse momentum $p_{T}$ for momentum cut-off parameter $\Delta p=0.3 \mathrm{GeV} / c$, at $\sqrt{s}=15 \mathrm{GeV}$ and $y=0$. 


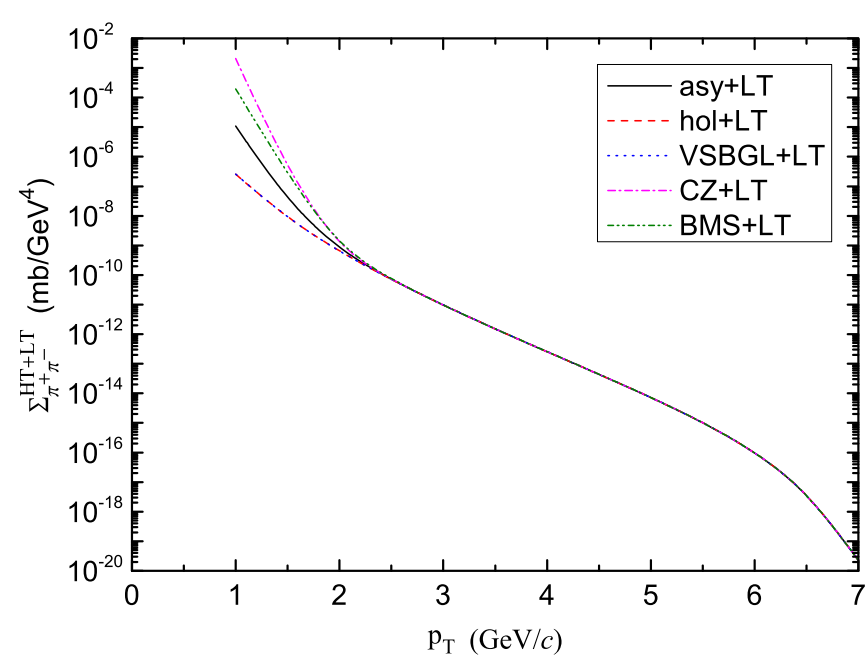

FIG. 4. The sum of HT and LT contribution to charged pion pair production $p \bar{p} \rightarrow \pi^{+} \pi^{-} X$ cross section $\Sigma_{\pi^{+}+\pi^{-}}^{\mathrm{HT}}$ as a function of the transverse momentum $p_{T}$ for momentum cut-off parameter $\triangle p=0.3 \mathrm{GeV} / c$, at $\sqrt{s}=15 \mathrm{GeV}$ and $y=0$. Notice that curves for asy, hol, VSBGL, CZ, and BMS pion DA in the region $2 \mathrm{GeV} / c<$ $p_{T}<7 \mathrm{GeV} / c$ completely overlap.

DA decreases from $2.11018 \times 10^{-3} \mathrm{mb} / \mathrm{GeV}^{4}$ to $2.26384 \times$ $10^{-20} \mathrm{mb} / \mathrm{GeV}^{4}$. From these results one can observe that HT cross section of the pion pair production in the protonantiproton collisions appears in the range and should be observable at the PANDA experiment.

In Fig. 5, we show the ratio $\Sigma_{\pi^{+} \pi^{-}}^{\mathrm{HT}} / \Sigma_{\pi^{+} \pi^{-}}^{\mathrm{LT}}$ for the process $p \bar{p} \rightarrow \pi^{+} \pi^{-} X$ as a function of $p_{T}$ for the pion DAs given in Eqs. (3.12)-(3.16) at $y=0$. It is seen that in the region $1 \mathrm{GeV} / c<p_{T}<3 \mathrm{GeV} / c$, the ratio $\Sigma_{\pi^{+} \pi^{-}}^{\mathrm{HT}} / \Sigma_{\pi^{+} \pi^{-}}^{\mathrm{LT}}$ for $\Phi^{\text {hol }}(x)$ is enhanced by about one order of magnitude relative to the $\Phi_{\mathrm{VSBGL}}(x)$. However, the enhancement are half an order of magnitude for $\Phi_{\mathrm{BMS}}\left(x, Q^{2}\right)$ and $\Phi_{\mathrm{CZ}}\left(x, Q^{2}\right)$, but in

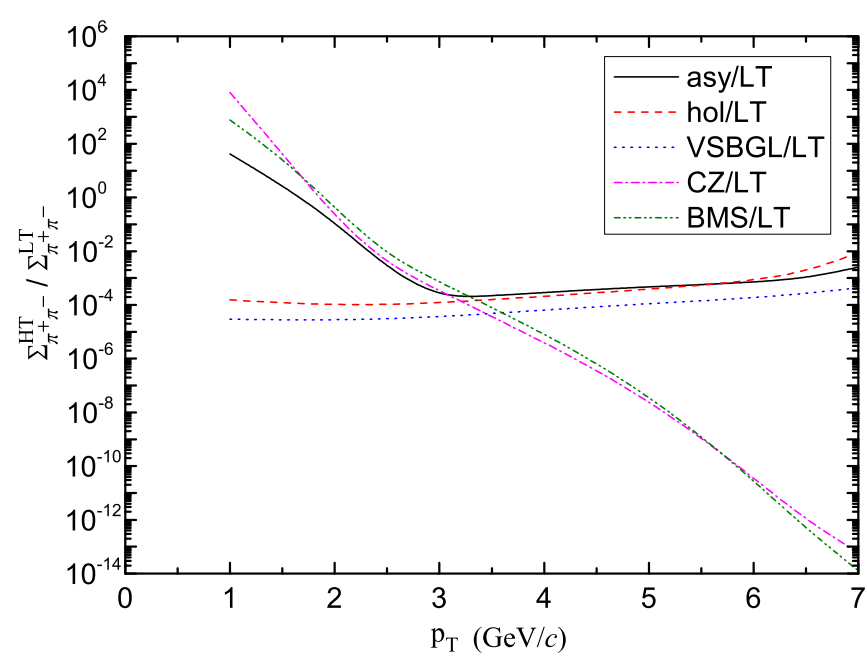

FIG. 5. Ratio $\Sigma_{\pi^{+} \pi^{-}}^{\mathrm{HT}} / \Sigma_{\pi^{+} \pi^{-}}^{\mathrm{LT}}$ as a function of the transverse momentum $p_{T}$ of the pion pair for $\left\langle q_{T}^{2}\right\rangle=0.25 \mathrm{GeV}^{2} / c^{2}$, at the c.m. energy $\sqrt{s}=15 \mathrm{GeV}$ and $y=0$.

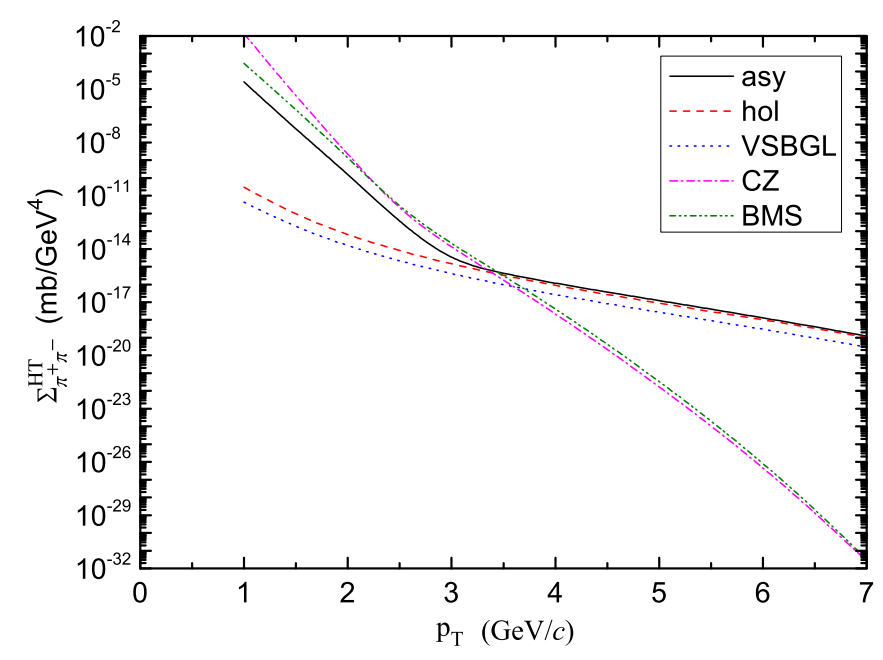

FIG. 6. HT contribution to charged pion pair production $p \bar{p} \rightarrow$ $\pi^{+} \pi^{-} X$ cross section $\Sigma_{\pi^{+} \pi^{-}}^{\mathrm{HT}}$ as a function of the transverse momentum $p_{T}$ for momentum cut-off parameter $\Delta p=0.3 \mathrm{GeV} / c$, at $\sqrt{s}=20 \mathrm{GeV}$ and $y=0$.

the region $3 \mathrm{GeV} / c<p_{T}<7 \mathrm{GeV} / c$ the magnitude relative for $\Phi^{\text {hol }}(x)$ and $\Phi_{\text {asy }}(x)$ pion distribution amplitudes is equal.

Through Figs. 6-8, we have displayed the $\Sigma_{\pi^{+} \pi^{-}}^{\mathrm{HT}}$, and $\Sigma_{\pi^{+} \pi^{-}}^{\mathrm{HT}+\mathrm{LT}}$ cross sections and the ratio $\Sigma_{\pi^{+} \pi^{-}}^{\mathrm{HT}} / \Sigma_{\pi^{+} \pi^{-}}^{\mathrm{LT}}$ which are calculated in the context of the FCC approach as a function of the pion pair transverse momentum $p_{T}$ for the pion DAs for Eqs. (3.12)-(3.16), and again for $y=0$ and at the centerof-mass energy $\sqrt{s}=20 \mathrm{GeV}$. It is seen from Figs. 6 and 8 that the $\Sigma_{\pi^{+} \pi^{-}}^{\mathrm{HT}}$, and $\Sigma_{\pi^{+} \pi^{-}}^{\mathrm{HT}+\mathrm{LT}}$ cross sections are monotonically decreasing with an increase in the transverse momentum of the pion pair. In the region $1 \mathrm{GeV} / c<p_{T}<7 \mathrm{GeV} / c$, the frozen cross section of the process $p \bar{p} \rightarrow \pi^{+} \pi^{-} X$ decreases

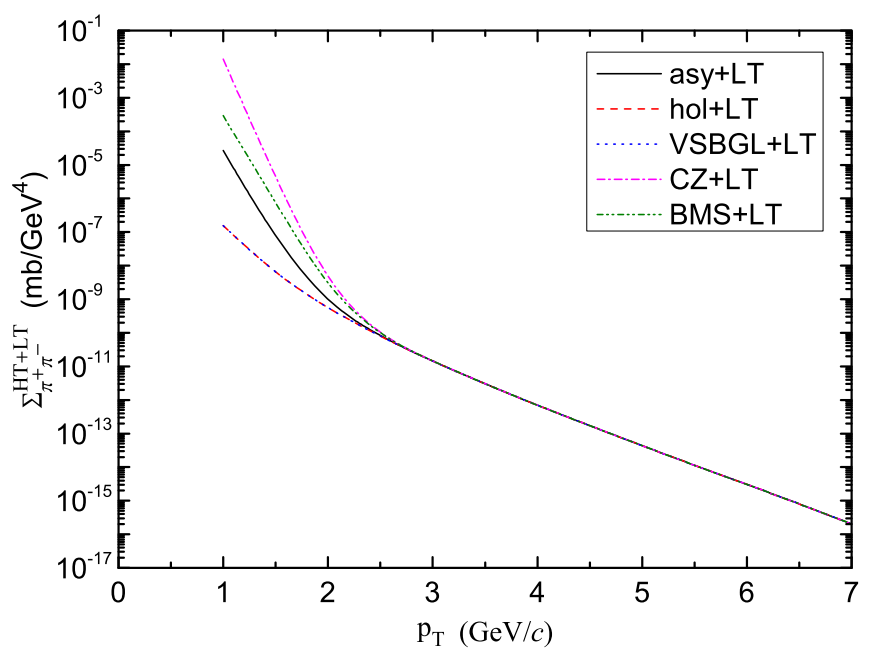

FIG. 7. The sum of HT and LT contribution to charged pion pair production $p \bar{p} \rightarrow \pi^{+} \pi^{-} X$ cross section $\Sigma_{\pi^{+}+\pi^{-}}^{\mathrm{HT}+\mathrm{LT}}$ as a function of the transverse momentum $p_{T}$ for momentum cut-off parameter $\Delta p=0.3 \mathrm{GeV} / c$, at $\sqrt{s}=20 \mathrm{GeV}$ and $y=0$. Notice that curves for asy, hol, VSBGL, CZ, and BMS pion distribution amplitudes in the region $2.5 \mathrm{GeV} / c<p_{T}<7 \mathrm{GeV} / c$ completely overlap. 


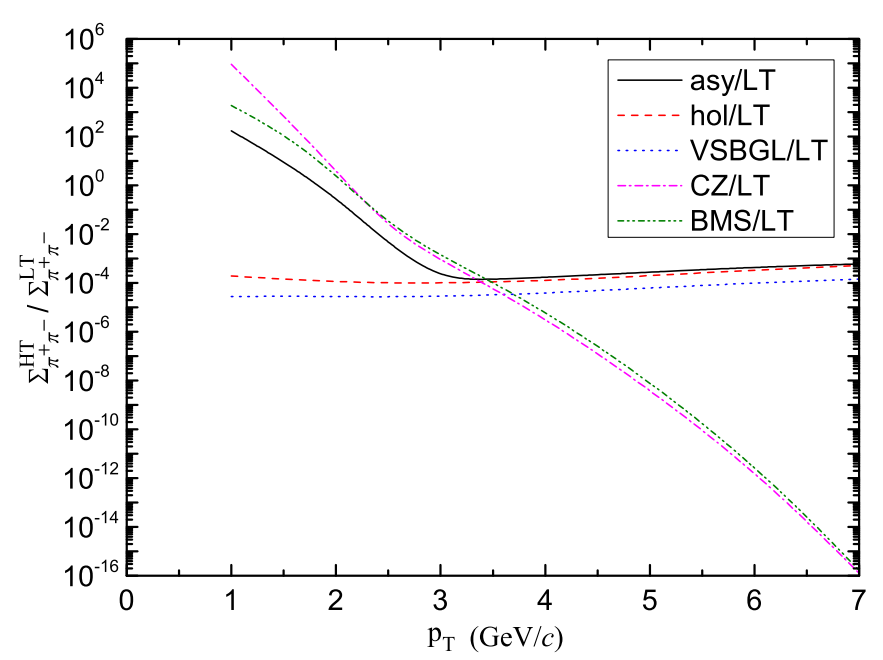

FIG. 8. Ratio $\Sigma_{\pi^{+} \pi^{-}}^{\mathrm{HT}} / \Sigma_{\pi^{+} \pi^{-}}^{\mathrm{LT}}$ as a function of the transverse momentum $p_{T}$ of the pion pair at the $\left\langle q_{T}^{2}\right\rangle=0.25 \mathrm{GeV}^{2} / c^{2}$, at the c.m. energy $\sqrt{s}=20 \mathrm{GeV}$ and $y=0$.

from $1.41213 \times 10^{-2} \mathrm{mb} / \mathrm{GeV}^{4}$ to $1.107 \times 10^{-19} \mathrm{mb} / \mathrm{GeV}^{4}$, but the sum of HT and LT cross section decreases from $1.41214 \times 10^{-2} \mathrm{mb} / \mathrm{GeV}^{4}$ to $2.01712 \times 10^{-16} \mathrm{mb} / \mathrm{GeV}^{4}$.

For the region $1 \mathrm{GeV} / c<p_{T}<4 \mathrm{GeV} / c$, the LT cross section is enhanced by about four orders of magnitude relative to the HT cross section calculated in the FCC approach. However, the $4 \mathrm{GeV} / c<p_{T}<7 \mathrm{GeV} / c$ region with increasing transverse momentum of the pair pion cross section increases, and the difference between leading and HT cross sections decreases essentially. Through Figs. 9-11, the dependence of the $\Sigma_{\pi^{+} \pi^{-}}^{\mathrm{HT}}$ and $\Sigma_{\pi^{+} \pi^{-}}^{\mathrm{HT}+\mathrm{LT}}$ cross sections and the ratio $\Sigma_{\pi^{+} \pi^{-}}^{\mathrm{HT}} / \Sigma_{\pi^{+} \pi^{-}}^{\mathrm{LT}}$ of the center-of-mass energy $\sqrt{s}$ for the pion DAs are displayed by using Eqs. (3.12)-(3.16) at $y=0$. Hereby, these figures indicate that the HT, sum of HT and LT cross sections, and the ratio increase slowly and smoothly when increasing the beam energy from $15 \mathrm{GeV}$ to $20 \mathrm{GeV}$ for each pion DAs.

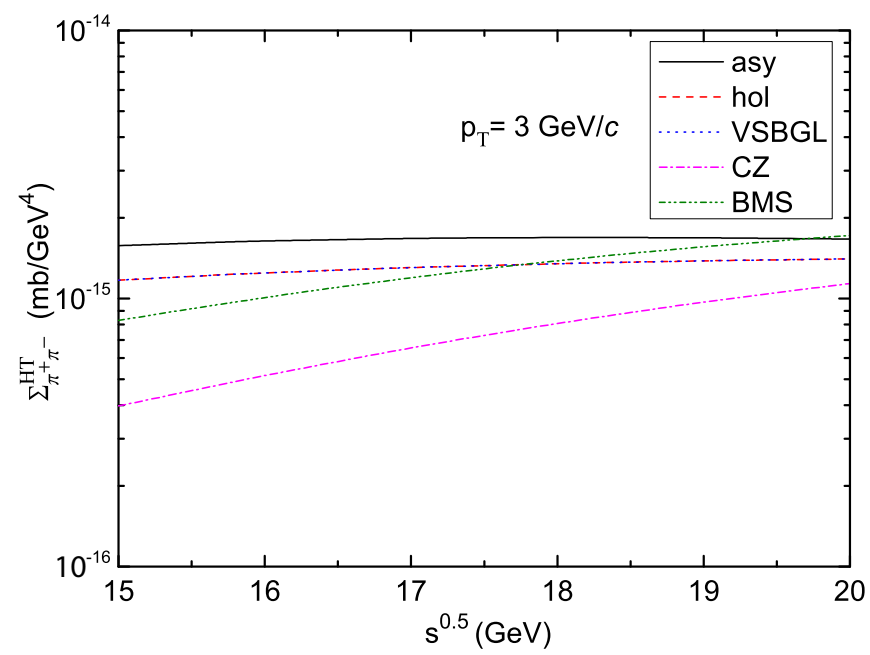

FIG. 9. HT contribution to charged pion pair production $p \bar{p} \rightarrow$ $\pi^{+} \pi^{-} X$ cross section $\Sigma_{\pi^{+} \pi^{-}}^{\mathrm{HT}}$ as a function of the center-of-mass energy $\sqrt{s}$ at the $\left\langle q_{T}^{2}\right\rangle=0.25 \mathrm{GeV}^{2} / c^{2}$ and $y=0$.

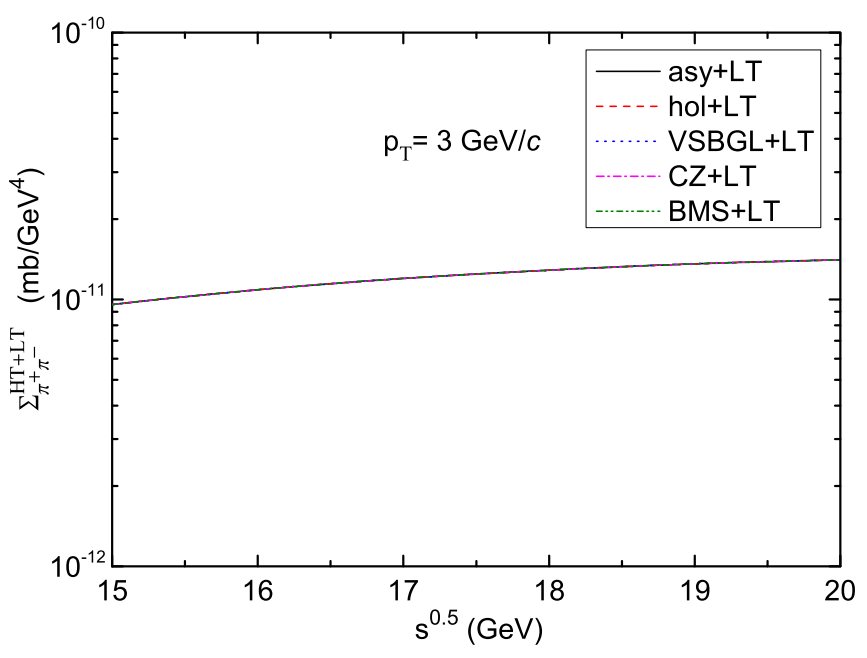

FIG. 10. The sum of HT and LT contribution to charged pion pair production $p \bar{p} \rightarrow \pi^{+} \pi^{-} X$ cross section $\Sigma_{\pi^{+}+\pi^{-}}^{\mathrm{HT}+\mathrm{LT}}$ as a function of the center-of-mass energy $\sqrt{s}$ for momentum cut-off parameter $\Delta p=0.3 \mathrm{GeV} / c$ and $y=0$. Notice that curves for asy, hol, VSBGL, $\mathrm{CZ}$, and BMS pion distribution amplitudes completely overlap.

In Fig. 12 we show that the ratio HT cross section $\Sigma_{\pi^{+} \pi^{-}}^{\mathrm{HT}}$ is calculated with $\left\langle q_{T}^{2}\right\rangle=0.25 \mathrm{GeV}^{2} / c^{2}$ and $\left\langle q_{T}^{2}\right\rangle=1 \mathrm{GeV}^{2} / c^{2}$ as a function of the pion pair transverse momentum $p_{T}$ for the pion DAs for Eqs. (3.12)-(3.16), at $y=0$ and the center-ofmass energy $\sqrt{s}=15 \mathrm{GeV}$.

One can also observe that the HT cross section in the region $1 \mathrm{GeV} / c<p_{T}<3 \mathrm{GeV} / c$ decreases more quickly for the DAs of asy, CZ, BMS with increasing $p_{T}$, but in the region $1 \mathrm{GeV} / c<p_{T}<7 \mathrm{GeV} / c$ increases more slowly and smoothly for the DAs hol, VSBGL with increasing $p_{T}$. In Figs. 13 and 14, the comparison of the HT cross section $\Sigma^{\mathrm{HT}}$ is displayed for the proton-antiproton annihilations into charged pion pairs $p \bar{p} \rightarrow \pi^{+} \pi^{-}$and elastic scattering $\pi p \rightarrow$ $\pi p$ processes which are calculated in the context of the FCC approach as a function of the pion pair transverse momentum

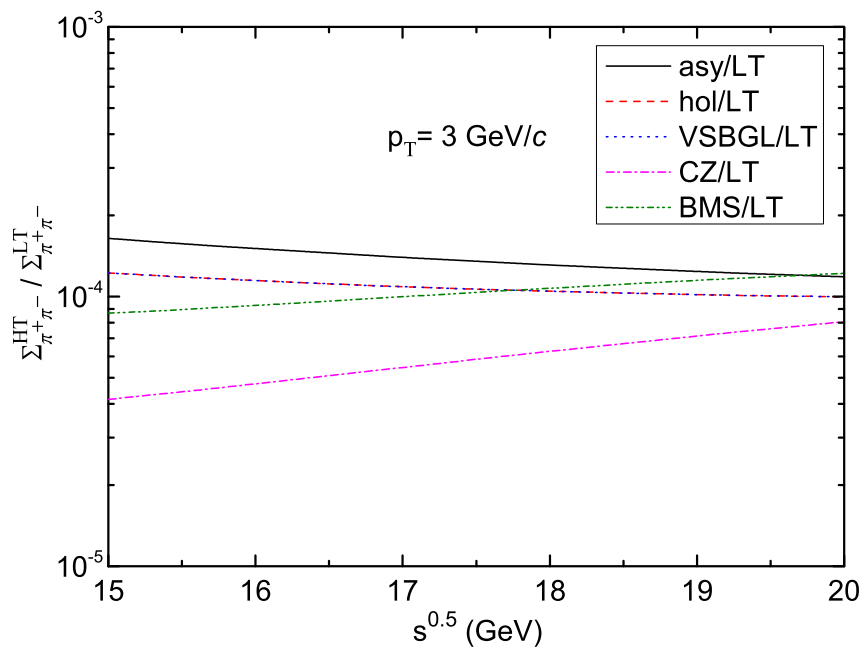

FIG. 11. Ratio $\Sigma_{\pi^{+} \pi^{-}}^{\mathrm{HT}} / \Sigma_{\pi^{+} \pi^{-}}^{\mathrm{LT}}$ as a function of the center-of-mass energy $\sqrt{s}$ at $\left\langle q_{T}^{2}\right\rangle=0.25 \mathrm{GeV}^{2} / c^{2}$ and $y=0$. 


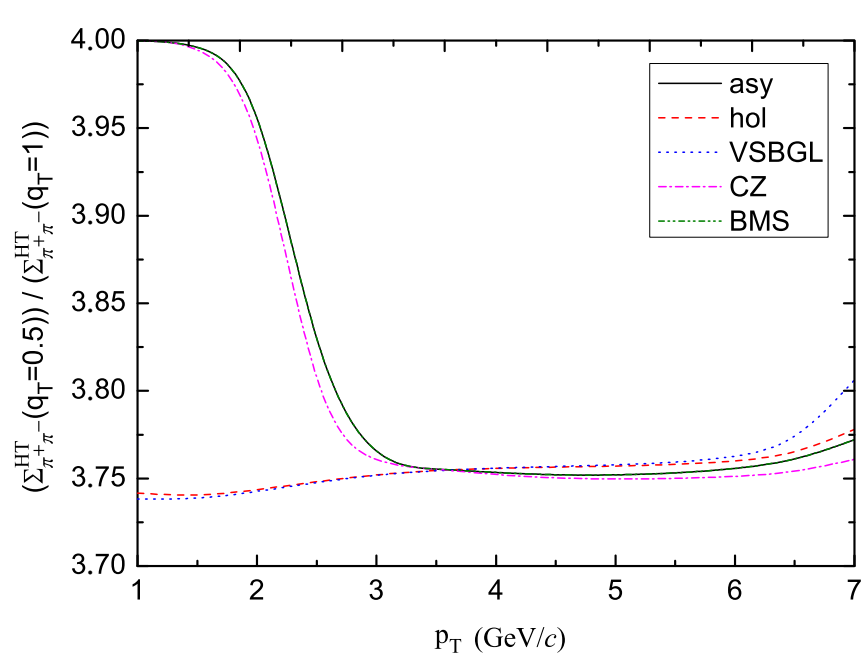

FIG. 12. Ratio HT cross sections $\Sigma_{\pi^{+} \pi^{-}}^{\mathrm{HT}}$ calculated with $\left\langle q_{T}^{2}\right\rangle=$ $0.25 \mathrm{GeV}^{2} / c^{2}$ and $\left\langle q_{T}^{2}\right\rangle=1 \mathrm{GeV}^{2} / c^{2}$ as a function of the pion pair transverse momentum $p_{T}$ at $\sqrt{s}=15 \mathrm{GeV}$ and $y=0$.

$p_{T}$ for the pion DAs at $y=0$ and the center-of-mass energy $\sqrt{s}=15 \mathrm{GeV}$. We can see from Figs. 13 and 14 that the HT cross section of the elastic scattering $\pi p \rightarrow \pi p$ process is enhanced by about half an order of magnitude relative to the $p \bar{p} \rightarrow \pi^{+} \pi^{-}$cross sections for all pion DAs.

In Figs. 15 and 16, we have displayed the HT and ratio HT to LT cross sections with the dependence on the variable $x_{T}$ ranging from $10^{-1}$ to 0.9 at the $p_{T}=6 \mathrm{GeV} / c$ with rapidities of pions $y_{1}=y_{2}=0$ for momentum cut-off parameter $\Delta p=0.5 \mathrm{GeV} / c$. As is seen from Fig. 15, the HT cross section in the region $0.1<x_{T}<0.4$ is monotonically increasing with an increase in the variable $x_{T}$. Approximately, the HT cross section for all DAs has a maximum at the point $x_{T}=0.4$. After this, the HT cross section with increasing $x_{T}$

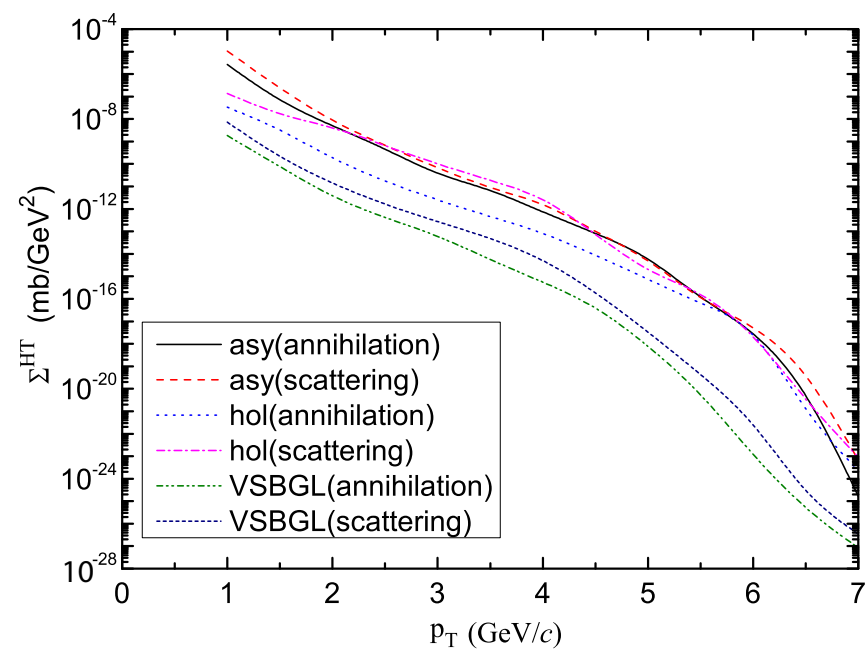

FIG. 13. HT $p \bar{p} \rightarrow \pi^{+} \pi^{-}$pion pair production and $\pi p \rightarrow \pi p$ cross sections as a function of the transverse momentum $p_{T}$ of the pion for $\left\langle q_{T}^{2}\right\rangle=0.25 \mathrm{GeV}^{2} / c^{2}$, at the c.m. energy $\sqrt{s}=15 \mathrm{GeV}$ and $y=0$.

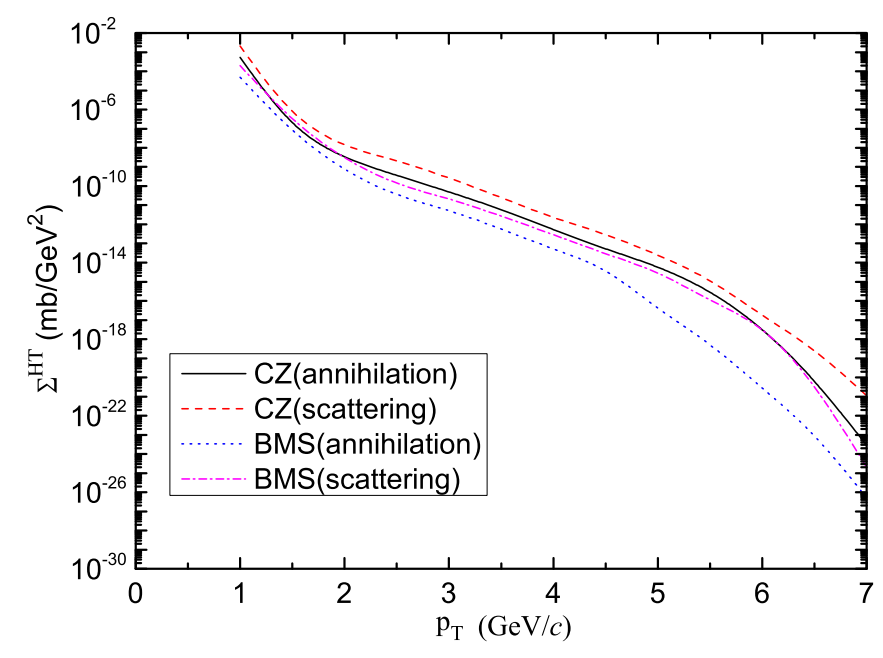

FIG. 14. HT $p \bar{p} \rightarrow \pi^{+} \pi^{-}$pion pair production and $\pi p \rightarrow \pi p$ cross sections as a function of the transverse momentum $p_{T}$ of the pion for $\left\langle q_{T}^{2}\right\rangle=0.25 \mathrm{GeV}^{2} / c^{2}$, at the c.m. energy $\sqrt{s}=15 \mathrm{GeV}$ and $y=0$.

is decreasing. But the ratio of HT to LT cross sections for the dependence on the variable $x_{T}$ has a different distinctive behavior. As is seen from Fig. 16, the ratio for the $\Phi_{\mathrm{CZ}}\left(x, Q^{2}\right)$ and $\Phi_{\mathrm{BMS}}\left(x, Q^{2}\right)$ has two minima and one maximum. The analysis of our calculations shows that the main reason for this depends on the phenomenological factors. These plots reveal that the distribution of variable $x_{T}$ also demonstrates the same dominant contributions in view of DAs as the ones in the transverse momentum dependence of the cross section. The ratio of HT to LT contributions remains almost nonstable in a large interval of $x_{T}$. This means that the ratio is more sensitive according to varying $x_{T}$. Analysis of our calculations shows that the HT cross section $\Sigma_{\pi^{+} \pi^{-}}^{\mathrm{HT}}$ and the ratio $\Sigma_{\pi^{+} \pi^{-}}^{\mathrm{HT}} / \Sigma_{\pi^{+} \pi^{-}}^{\mathrm{LT}}$ are sensitive to pion DA as predicted in the holographic and pQCD.

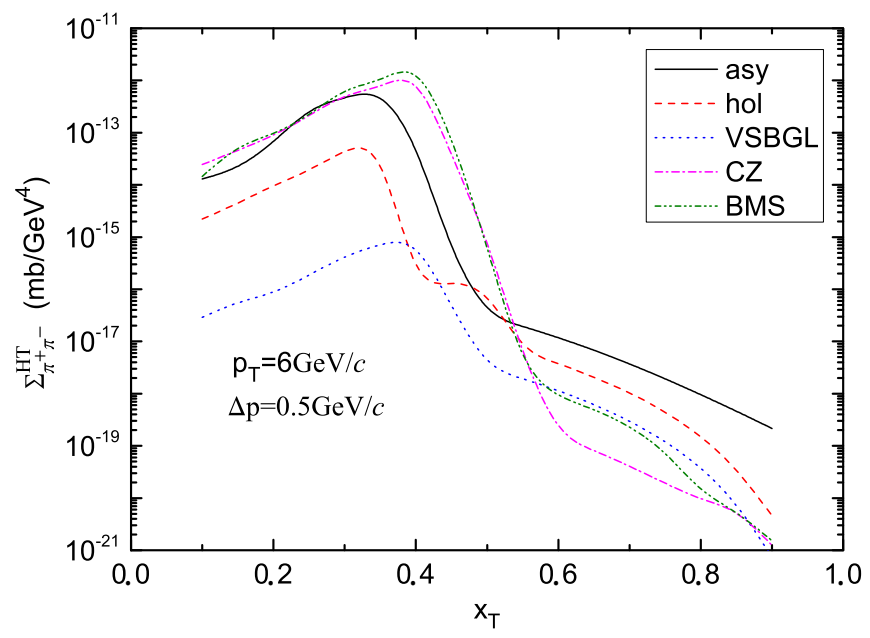

FIG. 15. HT $p \bar{p} \rightarrow \pi^{+} \pi^{-}$pion pair production cross section as a function of the variable $x_{T}$ for momentum cut-off parameter $\Delta p=$ $0.5 \mathrm{GeV} / c$ at $p_{T}=6 \mathrm{GeV} / c$ and $y=0$. 


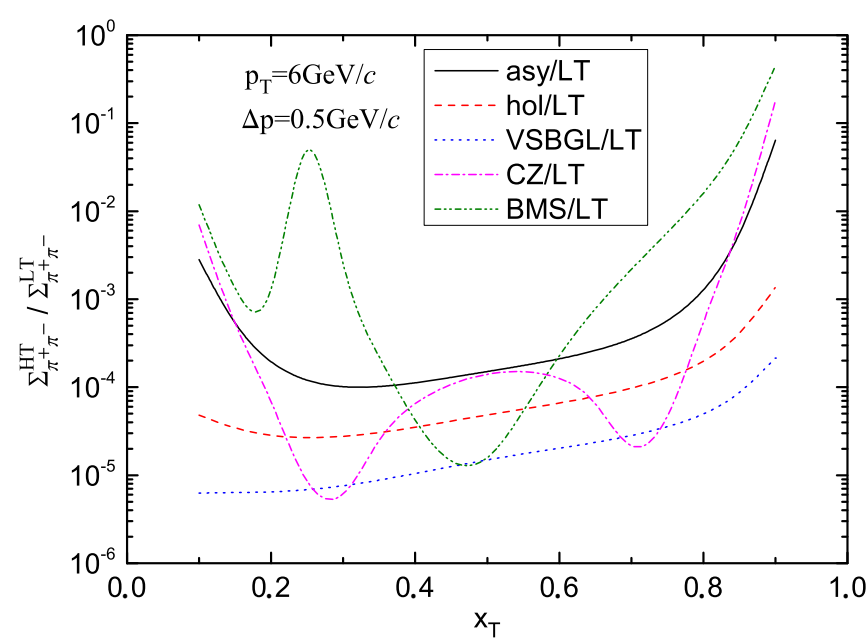

FIG. 16. Ratio of HT to LT contributions as a function of the variable $x_{T}$ for momentum cut-off parameter $\Delta p=0.5 \mathrm{GeV} / c$ at $p_{T}=6 \mathrm{GeV} / c$ and $y=0$.

\section{CONCLUSIONS}

In this study, the HT contributions, which are included in the direct and semidirect productions of the hard scattering process, to large- $p_{T}$ pion pair production in proton-antiproton collisions were discussed in detail. Furthermore, the dependence of HT contributions on pion-DAs predicted by the lightcone formalism and the light-front holographic AdS/CFT approach was addressed as well. It can be also concluded that the results which significantly depend on the DAs of the pion can be used for their research. The basic size of the HT cross sections was different depending on the choice of DAs of the produced pions and also some other phenomenological factors. Also, for the region $1 \mathrm{GeV} / c<p_{T}<3 \mathrm{GeV} / c$ DAs of $\mathrm{CZ}$, BMS, in the region $3 \mathrm{GeV} / c<p_{T}<7 \mathrm{GeV} / c$ hol, VSBGL gave the result which is close in shape to those for the asymptotic DA, but the HT contributions for $\mathrm{CZ}$ were larger than them by one order of magnitude relative of the asy and 2-3 orders for other DA. However, the ratio of HT to LT contributions allowed us to determine these regions in the phase space where HT contributions are essentially observable. This ratio is sensitive to the transverse momentum $p_{T}$ and the momentum cut-off parameter $\Delta p$, which is the detection limit for accompanying particles. For a small value of $p_{T}$, HT contributions yield the considerably high values. Its effect became significant at the small $p_{T}$ region compared to the LT contribution. It should be noted that semidirect pion pair production and double jet fragmentation to pion pair cross section strongly depend on the fragmentation function of the quark and gluon to pion. Also, the production of hadrons with large transverse momentum was dominated by the fragmentation of partons which is produced in parton-parton scattering with large momentum. The production cross section for this hard scattering depends on the initial distribution of partons in the colliding species, the elementary parton-parton cross section, and the fragmentation process of partons into hadrons.

The HT cross section obtained in our study should be observable at a hadron collider. Also, the feature of HT effects can help theoretical interpretations of the future PANDA experimental data for the direct inclusive pion pair production cross section in the proton-antiproton collisions. As a result, it can be indicated that the HT processes for large- $p_{T}$ pion pair production have a key enabling contribution, where the pions are generated directly in the hard-scattering subprocess, rather than by gluon and quark fragmentation. Inclusive pion pair production provides an essential test case where HT contributions dominate those of LT in the certain kinematic regions. The HT contributions can be utilized to interpret theoretically the future experimental data for the charged pion pair production in $p \bar{p}$ collisions. The results of this work can be useful to provide a simple test of the short distance structure of QCD as well as to determine more precise DAs of the pion.

\section{ACKNOWLEDGMENTS}

A.I.A. is grateful for the financial support by the Science Development Foundation under the President of the Republic of Azerbaijan - Grant No. EIF/MQM/Elm-Tehsil-1-20161(26)-71/11/1 and Baku State University Grant No. " $50+50$ " (2018-2019). The authors are grateful to S. V. Mikhailov for useful discussions.
[1] G. P. Lepage and S. J. Brodsky, Phys. Lett. B 87, 359 (1979).

[2] A. V. Efremov and A. V. Radyushkin, Theor. Math. Phys. 42, 97 (1980).

[3] A. V. Efremov and A. V. Radyushkin, Phys. Lett. B 94, 245 (1980).

[4] V. M. Braun et al., Nucl. Phys. B 685, 171 (2004).

[5] V. M. Braun, arXiv:hep-ph/9801222.

[6] V. M. Braun, G. P. Korchemsky, and D. Muller, Prog. Part. Nucl. Phys. 51, 311 (2003).

[7] G. P. Lepage and S. J. Brodsky, Phys. Rev. D 22, 2157 (1980).

[8] P. Colangelo and A. Khodjamirian, arXiv:hep-ph/0010175.

[9] C. W. Bauer, D. Pirjol, and I. W. Stewart, Phys. Rev. D 67, 071502 (2003).
[10] M. Beneke and Th. Feldmann, Nucl. Phys. B 685, 249 (2004).

[11] S. J. Brodsky, G. P. Lepage, and P. B. Mackenize, Phys. Rev. D 28, 228 (1983).

[12] M. Mojaza, S. J. Brodsky, and X. G. Wu, Phys. Rev. Lett. 110, 192001 (2013).

[13] V. N. Gribov and L. N. Lipatov, Sov. J. Nucl. Phys. 15, 438 (1972).

[14] L. N. Lipatov, Sov. J. Nucl. Phys. 20, 94 (1975).

[15] Y. L. Dokshitzer, JETP 46, 641 (1977).

[16] G. Altarelli and G. Parisi, Nucl. Phys. B 126, 298 (1977).

[17] F. M. Dittes and A. V. Radyushkin, Phys. Lett. B 134, 359 (1984)

[18] M. H. Sarmadi, Phys. Lett. B 143, 471 (1984).

[19] G. R. Katz, Phys. Rev. D 31, 652 (1985). 
[20] S. V. Mikhailov and A. V. Radyushkin, Nucl. Phys. B 254, 89 (1985).

[21] F. Arleo, S. J. Brodsky, D. S. Hwang, and A. M. Sickles, Phys. Rev. Lett. 105, 062002 (2010).

[22] J. Collins and J.-W. Qiu, Phys. Rev. D 75, 114014 (2007).

[23] D. Boer, S. J. Brodsky, and D. S. Hwang, Phys. Rev. D 67, 054003 (2003).

[24] A. I. Ahmadov, I. Boztosun, R. Kh. Muradov, A. Soylu, and E. A. Dadashov, Int. J. Mod. Phys. E 15, 1209 (2006).

[25] A. I. Ahmadov, I. Boztosun, A. Soylu, and E. A. Dadashov, Int. J. Mod. Phys. E 17, 1041 (2008).

[26] A. I. Ahmadov, C. Aydin, Sh. M. Nagiyev, Y. A. Hakan, and E. A. Dadashov, Phys. Rev. D 80, 016003 (2009).

[27] A. I. Ahmadov, C. Aydin, E. A. Dadashov, and Sh. M. Nagiyev, Phys. Rev. D 81, 054016 (2010).

[28] A. I. Ahmadov and R. M. Burjaliyev, Int. J. Mod. Phys. E 20, 1243 (2011).

[29] A. I. Ahmadov, Sh. M. Nagiyev, and E. A. Dadashov, Int. J. Mod. Phys. E 21, 1250014 (2012).

[30] A. I. Ahmadov, C. Aydin, and F. Keskin, Phys. Rev. D 85, 034009 (2012).

[31] A. I. Ahmadov, C. Aydin, and F. Keskin, Ann. Phys. (NY) 327, 1472 (2012).

[32] A. I. Ahmadov, C. Aydin, and O. Uzun, Phys. Rev. D 87, 014006 (2013).

[33] A. I. Ahmadov, C. Aydin, and O. Uzun, Phys. Rev. D 89, 014018 (2014).

[34] J. A. Bagger and J. F. Gunion, Phys. Rev. D 29, 40 (1984).

[35] J. A. Bagger and J. F. Gunion, Phys. Rev. D 25, 2287 (1982).

[36] V. N. Baier and A. Grozin, Phys. Lett. B 96, 181 (1980).

[37] S. Gupta, Phys. Rev. D 24, 1169 (1981).

[38] F. S. Sadykhov and A. I. Akhmedov, Russ. Phys. J. 38, 513 (1995).

[39] M. Demirci and A. I. Ahmadov, Int. J. Mod. Phys. A 33, 1850166 (2018).

[40] C. R. Ji and F. Amiri, Phys. Rev. D 42, 3764 (1990).

[41] T. Buran, A. Eide, P. Helgaker, P. Lehmann, A. Lundby et al., Nucl. Phys. B 116, 51 (1976).

[42] W. Ying, J. Phys.: Conf. Ser. 742, 012021 (2016).

[43] R. Shyam and K. Tsushima, Phys. Rev. D 94, 074041 (2016).

[44] Y. Wang, Yu. M. Bystritskiy, and E. Tomasi-Gustafsson, Phys. Rev. C 95, 045202 (2017).

[45] Y. Wang, Y. M. Bystritskiy, A. I. Ahmadov, and E. TomasiGustafsson, Phys. Rev. C 96, 025204 (2017).

[46] L. A. Harland-Lang, V. A. Khoze, M. G. Ryskin, and W. J. Stirling, Eur. Phys. J. C 71, 1714 (2011).

[47] K. Djagouri, J. J. Dugne, C. Carimalo, and P. Kessler, Z. Phys. C 45, 267 (1989).
[48] W. Erni et al. (PANDA Collaboration), arXiv:0903.3905.

[49] A. V. Luchinsky and S. V. Poslavsky, Phys. Rev. D 85, 074016 (2012).

[50] R. Baier, J. Engels, and B. Petersson, Z. Phys. C 2, 265 (1979).

[51] G. Curci, M. Greco, and Y. Srivastava, Phys. Rev. Lett. 43, 834 (1979).

[52] G. Curci, M. Greco, and Y. Srivastava, Nucl. Phys. B 159, 451 (1979).

[53] M. Greco, Phys. Lett. B 100, 351 (1981).

[54] M. Greco, Y. Srivastava, and G. Penso, Phys. Rev. D 21, 2520 (1980).

[55] Y. I. Dokshitzer and B. R. Webber, Phys. Lett. B 404, 321 (1997).

[56] Y. I. Dokshitzer, G. Marchesini, and B. R. Webber, Nucl. Phys. B 469, 93 (1996).

[57] S. Mandelstam, Phys. Rev. D 20, 3223 (1979).

[58] N. Brown and M. R. Pennington, Phys. Rev. D 38, 2266 (1988).

[59] N. Brown and M. R. Pennington, Phys. Rev. D 39, 2723 (1989).

[60] J. M. Cornwall, Phys. Rev. D 26, 1453 (1982).

[61] R. Alkofer and L. von Smekal, Phys. Rep. 353, 281 (2001).

[62] L. von Smekal, A. Hauck, and R. Alkofer, Ann. Phys. (NY) 267, 1 (1998).

[63] G. Parisi and R. Petronzio, Phys. Lett. B 94, 51 (1980).

[64] J. Schwinger, Phys. Rev. 127, 324 (1962).

[65] J. F. Owens, Rev. Mod. Phys. 59, 465 (1987).

[66] J. F. Gunion and B. Petersson, Phys. Rev. D 22, 629 (1980).

[67] C. Carimalo, M. Crozon, P. Kessler, and J. Parisi, Phys. Rev. D 30, 576 (1984).

[68] B. A. Kniehl, G. Kramer, and B. Potter, Nucl. Phys. B 582, 514 (2000).

[69] S. J. Brodsky and G. L. Lepage, in Perturbative Quantum Chromodynamics, edited by A. H. Mueller (World Scientific, Singapore, 1989), p. 93.

[70] S. J. Brodsky, H.-C. Pauli, and S. S. Pinsky, Phys. Rep. 301, 299 (1998).

[71] V. L. Chernyak and A. R. Zhitnitsky, Phys. Rep. 112, 173 (1984).

[72] A. Vega, I. Schmidt, T. Branz, T. Gutsche, and V. E. Lyubovitskij, Phys. Rev. D 80, 055014 (2009).

[73] S. J. Brodsky and G. F. de Teramond, Phys. Rev. D 77, 056007 (2008).

[74] S. J. Brodsky, Proc. Sci. LHC07, 002 (2007).

[75] A. P. Bakulev, S. V. Mikhailov, and N. G. Stefanis, Phys. Lett. B 578, 91 (2004); A. P. Bakulev, S. V. Mikhailov, A. V. Pimikov, and N. G. Stefanis, Phys. Rev. D 86, 031501 (2012).

[76] A. D. Martin, W. J. Stirling, R. S. Thorne, and G. Watt, Eur. Phys. J. C 63, 189 (2009). 